\title{
Versão Infantojuvenil do Inventário de Percepção de Suporte Familiar (IPSF-IJ)
}

\author{
Makilim Nunes Baptista (D) \\ Universidade São Francisco, Campinas-SP, Brasil \\ Katya Luciane de Oliveira (D) \\ Universidade Estadual de Londrina, Londrina-PR, Brasil \\ Amanda Lays Monteiro Inácio ${ }^{1}$ (D) \\ Universidade São Francisco, Campinas-SP, Brasil \\ Marcella das Neves Burgos (D) \\ Universidade Estadual de Londrina, Londrina-PR, Brasil \\ Evandro Morais Peixoto (D) \\ Universidade São Francisco, Campinas-SP, Brasil
}

RESUMO

Este estudo teve por objetivo estimar evidências de validade com base na estrutura interna e precisão do Inventário de Percepção de Suporte Familiar (IPSF) em crianças e adolescentes e, obter uma versão breve do instrumento. Participaram, respectivamente, 355 alunos $(M=12,6 ; D P=1,2)$ e 453 alunos $(M=12,3 ; D P=1,4)$ do $6^{\circ}$ ao $9^{\circ}$ ano do ensino fundamental público. A análise fatorial exploratória indicou estrutura composta por três fatores: Autonomia, Adaptação e Afetivo-Consistente, corroborando os resultados do IPSF adulto. Os índices de precisão, $\Omega$ entre 0,726 e 0,881 , complementaram as adequadas propriedades psicométricas dessa versão. A análise fatorial confirmatória foi realizada para a versão breve do IPSF-IJ, com 18 itens, indicando replicabilidade da estrutura interna e manutenção dos indicadores de precisão, $\Omega$ entre 0,806 e 0,878 . Conclui-se que ambas as versões do instrumento são medidas adequadas da percepção de suporte familiar em crianças e adolescentes.

Palavras-chave: criança; suporte familiar; avaliação psicológica; psicometria.

\section{ABSTRACT - Child-Adolescent Version of the Perception of Family Support Inventory}

This study aimed to estimate evidence of validity based on the internal structure and accuracy of the Inventário de Percepção de Suporte Familiar (IPSF - Perception of Family Support Inventory) in children and adolescents and to obtain a brief version of the instrument. Participants were, respectively, 355 students $(M=12.6 ; S D=1.2)$ and 453 students $(M=12.3 ; S D=1.4)$ of the 6 th to 9th years of public elementary school. The exploratory factor analysis indicated a structure composed of three factors: Autonomy, Adaptation and Affective-Consistent, corroborating the results of the adult IPSF. Accuracy indices, $\Omega$ between 0.726 and 0.881 , complemented the adequate psychometric properties of this version. Confirmatory factor analysis was performed for the short version of the IPSF-IJ, with 18 items, indicating replicability of the internal structure and maintenance of the accuracy indicators, $\Omega$ between 0.806 and 0.878 . It was concluded that both versions of the instrument are adequate measures of the perception of family support in children and adolescents.

Keywords: child; family support; psychological evaluation, psychometry.

RESUMEN - Versión Infantil y Juvenil del Inventario de Percepción de Apoyo Familiar (IPSF-IJ)

Este estudio tuvo como objetivo estimar evidencias de validez en función de la estructura interna y la precisión del Inventario de Percepción de Apoyo familiar (IPSF) en niños y adolescentes y obtener una versión breve del instrumento. Participaron, respectivamente, 355 estudiantes $(\underline{X}=12.6 ; D S=1.2)$ y 453 estudiantes $(\underline{X}=12.3 ; D S=1.4)$ del sexto al noveno año de la educación primaria pública. El análisis factorial exploratorio indicó una estructura compuesta por tres factores: Autonomía, Adaptación y Afectivo Consistente, corroborando los resultados de la IPSF adulta. Los índices de precisión, $\Omega$ entre 0.726 y 0.881 , complementaron las propiedades psicométricas apropiadas de esta versión. Se realizó un análisis factorial confirmatorio para la versión corta del IPSF-IJ, con 18 ítems, resultando en la replicabilidad de la estructura interna y el mantenimiento de indicadores de precisión, $\Omega$ entre 0.806 y 0.878. Se concluye que ambas versiones del instrumento son adecuadas para la percepción del apoyo familiar en niños y adolescentes. Palabras clave: Niños; apoyo familiar; evaluación psicológica, psicometría.

A criança inicia seu desenvolvimento social por meio de seus primeiros laços e relacionamentos estabelecidos no seio familiar. Enquanto isso, os adultos servem como paradigma e suporte para que ela se

1 Endereço para correspondência: Departamento de Psicologia e Psicanálise. Centro de Ciências Biológicas. Universidade Estadual de Londrina, Campus Universitário. Rodovia Celso Garcia Cid (PR 445), km 380, Cx Postal 6.001, 86051-990, Londrina, PR. Tel.: (43) 3371-4397. E-mail: amandalmonteiroo@gmail.com Artigo derivado da Dissertação de mestrado de Marcela das Neves Burgos com orientação de Katya Luciane de Oliveira, defendida em 2018 no programa de pós-graduação em Educação da Universidade Estadual de Londrina. 
desenvolva integralmente (Ribeiro, Ciasca, \& Capelatto, 2016). Segundo Guidetti e Martinelli, (2017), o ambiente familiar deve oferecer estabilidade emocional, fazendo-se necessário que a criança perceba o apoio e suporte oferecidos pela família, pois essa percepção é capaz de afetar as relações familiares e sociais, seu contexto de aprendizagem e ainda o desenvolvimento da personalidade.

A expressão das interações entre os membros da família correspondem a manifestação de fatores, como atenção, cuidado, afeto, proteção, autonomia, carinho, comunicação e outros (Baptista, 2009; Pozzobon \& Marin, 2017). O suporte advém do contato entre familiares a fim de promover bem-estar, influenciando positivamente os vínculos e as emoções dos membros da família. Também tem o papel de estabelecer modelos de relações, transmitindo-os a todos os familiares, de modo que estes possam desenvolver a capacidade de transpor obstáculos do cotidiano (Costa, Montiel, Bartholomeu, Murgo, \& Campos, 2016).

O suporte familiar é uma base primária primordial para que ocorra a socialização, por meio da transmissão de padrões comportamentais, como valores, linguagem, crenças e outros. Ademais, funciona de forma recíproca, pois gera efeitos, como afeição e formação social, tanto para quem recebe, quanto para quem os oferece (Batista, Mantovani, \& Nascimento, 2015; Baptista, Rueda, \& Brandão, 2017). Nessa mesma linha, o suporte familiar é constituído pela coesão estabelecida na ligação entre os integrantes de uma família. Essa ligação é capaz de promover a autonomia e a adaptabilidade familiar. Por sua vez, a adaptação familiar diz respeito à capacidade da família em se adaptar às mudanças que podem interferir nos princípios e sentimentos expressos (Baptista, 2005; Batista et al., 2015).

A percepção de um suporte familiar satisfatório permite a prevenção de fatores relacionados a distúrbios afetivos de adultos, adolescentes e crianças (Gonçalves, Baptista, \& Farcas, 2016). A literatura científica também indica que o suporte familiar é um fator capaz de intervir positivamente na autonomia e deleite de vida, possibilitando a diminuição de comportamentos negativos perante situações de estresse. Pode, também, interferir no autoconceito do indivíduo, ou seja, em suas crenças sobre si mesmo, de forma positiva quando adequado (Baptista et al., 2017; Bastos, Scortegagna, Baptista, \& Cremasco, 2016).

Por sua vez, um contexto familiar caracterizado por punição, falta de afeto e opressão, tende a prejudicar a autonomia e o desenvolvimento afetivo saudável do indivíduo, influenciando sobremaneira em diferentes instâncias de sua vida. Além disso, esses fatores podem ser compreendidos como associados à ansiedade e à depressão entre seus membros (Barbosa \& Wagner, 2013; Batista et al., 2015; Van Harmelen et al., 2016).

O suporte familiar percebido deve ser diferenciado do suporte recebido, uma vez que o modo como um indivíduo entende demonstrações de afeto, atenção, entre outros, pode ser diferente da maneira como outro membro da família capta essa mesma demonstração (Guidetti \& Martinelli, 2017). Assim, o suporte deve ser considerado como dinâmico, pois manifesta-se de maneiras diversas a depender também da percepção de cuidado daquele que o recebe. Nota-se que a valorização do suporte fornecido pela família, bem como do relacionamento intrafamiliar mostram ser recursos de grande importância para que a pessoa apresente percepção de bem-estar em relação à própria vida, autoestima e saúde mental (Castro \& Cruz, 2015).

Guidetti e Martinelli (2017) observaram que as percepções a respeito do suporte familiar de crianças e adolescentes costumam ser mais positivas quando seus pais expressam mais seus próprios sentimentos, motivações e pensamentos. Nessa mesma direção, Knight, Boden e Holt (2010) afirmam que crianças e adolescentes tendem a apresentar satisfação em todas as áreas de sua vida, quando percebem maior suporte emocional dos pais.

Autores como Batista, Mantovani e Nascimento (2015), Benetti, Vieira e Faracco (2016), Pozzobon e Marin (2017) e Roksa e Kinsley (2019) afirmam que o suporte familiar possui influência significativa no desenvolvimento das mais diversas potencialidades do indivíduo, sendo uma dessas vertentes o sucesso escolar. $\mathrm{O}$ envolvimento dos pais na vida escolar dos filhos tende a colaborar, não somente com o desempenho dos estudantes, como também com a criação de hábitos escolares positivos, realização de tarefas, gestão do tempo, entre outros, o que auxilia no desenvolvimento das competências importantes ao bom desempenho escolar. Contudo, os autores ressaltam que a ausência do papel exercido pelos familiares no ambiente escolar pode ser um dos fatores relacionados à baixa autoestima e dificuldades de aprendizagem.

O Inventário de Percepção de Suporte Familiar (IPSF) é um instrumento de medida construído e com evidências de validade para o Brasil para se mensurar o Suporte Familiar (Baptista, 2007, 2009). Foi desenvolvido e testado no que tange a estrutura interna e confiabilidade por Baptista (2005) em uma amostra de 346 estudantes universitários do interior de São Paulo, com idades entre 17 e 55 anos $(M=24,67 ; D P=6,36)$. Nesse estudo, a análise fatorial exploratória indicou a existência de quatro fatores, denominados como Inadaptação familiar, Afetividade familiar, Consistência familiar e Autonomia familiar, todos os itens com carga fatorial acima de 0,45 e a consistência interna calculada pelo alfa variou entre 0,81 e 0,88 .

Na sequência, Baptista (2007) buscou comparar os componentes do inventário em duas configurações de participantes, por meio de uma amostra de 1.064 estudantes provenientes do ensino médio e universitário do estado de São Paulo, com idades entre 17 e 64 anos $(M=23,35 ; D P=6,05)$. Nessa ocasião, o resultado da 
análise da estrutura interna evidenciou três dimensões, a saber, Afetivo-consistente $(\alpha=0,91)$, Adaptação $(\alpha=0,90)$, e Autonomia $(\alpha=0,78)$, além de um valor de 0,93 para o alfa da escala total. Todos os itens possuíram cargas fatoriais acima de 0,30 .

Por fim, o instrumento foi testado pelo mesmo autor em 2009 com uma amostra geral da população $(n=1204)$, com participantes com idades que variavam de 11 a 57 anos $(M=22,6 ; D P=7,1)$. Essa distribuição amostral, apesar de contemplar indivíduos do ensino fundamental, possui poucos sujeitos dessa etapa escolar em comparação com a amostra de adultos. Nessa versão final, o instrumento apresentou, novamente, uma estrutura organizada em três dimensões, todas com consistência interna aceitável fornecida pelo alfa de Cronbach. São elas: Afetivo-consistente $(\alpha=0,91)$, Adaptação $(\alpha=0,83)$, Autonomia $(\alpha=0,80)$ e um fator geral denominado IPSF total $(\alpha=0,93)$.

No que se refere aos estudos que fizeram uso desse instrumento, Costa, Montiel, Bartholomeu, Murgo e Campos (2016) avaliaram as relações entre o suporte familiar e o desempenho de leitura e escrita de 102 crianças provenientes do ensino fundamental do Maranhão, com idades entre 8 e 10 anos. Os resultados evidenciaram que as percepções positivas dos suportes social e familiar contribuíram para a motivação no processo de aquisição da leitura e escrita.

Guidetti e Martineli (2017) analisaram a percepção de alunos de diferentes anos escolares do ensino fundamental acerca da motivação escolar e do suporte. Participaram desse estudo 342 crianças matriculadas do $3^{\circ}$ ao $5^{\circ}$ ano do primeiro ciclo do ensino fundamental do município de São Paulo, com idades variando de 7 a 13 anos. Como resultados, observou-se que houve uma diminuição do suporte familiar à medida que o aluno progredia o ano escolar, bem como houve relação positiva e estatisticamente significativa entre a motivação para aprender e o suporte familiar recebido.

Diante do exposto, reitera-se a relevância do suporte familiar para os processos de aprendizagem, sobretudo no início da escolarização, e ainda a incipiência de estudos que busquem analisar essa variável com uma amostra que ofereça um parâmetro mais afinado com a realidade escolar. Posto isso, o presente estudo buscou estimar evidências de validade com base na estrutura interna e precisão do IPSF em uma amostra formada por escolares da segunda etapa do ensino fundamental, por intermédio da análise fatorial exploratória (AFE). Objetivou-se ainda a realização de uma análise fatorial confirmatória (AFC) em uma segunda amostra de escolares para uma versão abreviada do IPSF-IJ e o cálculo dos dados descritivos para ambas as amostras pesquisadas.

Para o desenvolvimento da versão breve, considerou-se as recomendações metodológicas sumarizadas por Maiano et al. (2008), a partir das sugestões de Marsh, Ellis, Parada, Richards e Heubeck (2005) e Smith, McCarthy e Anderson (2000). Essas recomendações apontam que o desenvolvimento de uma versão breve deve partir de uma versão longa consistente e ser testada em uma amostra independente, deve manter a cobertura do conteúdo avaliado na versão longa, replicar a estrutura fatorial da forma longa, conservar as propriedades de confiabilidade e equivalência de evidências de validade baseadas na relação com variáveis externas com a versão longa.

\section{Método}

\section{Participantes}

A primeira amostra foi selecionada por conveniência, sendo composta por 355 alunos matriculados nos $6^{\circ}$ $(25,6 \% ; n=91), 7^{\circ}(34,6 \%, n=123), 8^{\circ}(25,1 \%, n=89) \mathrm{e}$ $9^{\circ}(14,6 \%, n=52)$ anos do ensino fundamental II de duas escolas públicas estaduais do interior do estado do Paraná. A média de idade foi de 12 anos e 6 meses $(D P=1,2)$, sendo a mínima encontrada de 10 anos e a máxima de 16 anos. O sexo masculino representou 39\% $(n=139)$ da amostra e o feminino $60 \%(n=213)$, sendo que $1 \%(n=3)$ dos participantes não respondeu essa questão.

A segunda amostra foi selecionada por conveniência, sendo composta por 453 alunos matriculados nos $6^{\circ}$ (42,8\%; $n=194), 7^{\circ}(18,1 \%, n=82), 8^{\circ}(19,2 \%, n=87) \mathrm{e}$ $9^{\circ}(19,9 \%, n=90)$ anos do ensino fundamental II de duas escolas públicas estaduais do interior do estado do Paraná. A média de idade foi de 12 anos e 3 meses $(D P=1,4)$, a idade mínima foi de 10 anos e a máxima de 16 anos. O sexo feminino representou 52,5\% $(n=238)$ da amostra e o masculino $47,5 \%(n=215)$.

\section{Instrumento}

Foi utilizado o Inventário de Percepção de Suporte Familiar (IPSF) de Baptista (2009). O instrumento é constituído por 42 afirmações que abarcam situações familiares, em que a pessoa deve assinalar a frequência com que cada uma delas acontece em sua família. Os itens são distribuídos em três subescalas, sendo elas: Afetivoconsistente $(\alpha=0,91$; itens $2,3,4,5,9,11,15,17,22,23$, 24, 25, 26, 28, 29, 30, 31, 35, 37, 41 e 42); Adaptação, na qual as questões têm pontuação invertida $(\alpha=0,83$; itens $1,6,7,8,13,16,20,21,27,32,33,36$ e 39); Autonomia $(\alpha=0,80$; itens $10,12,14,18,19,34,38$ e 40). Também há a somatória total dos itens da escala, que corresponde ao IPSF total $(\alpha=0,93)$.

Conforme indica Baptista (2009), no inventário a subescala Afetivo-consistente se refere à expressão de afetividade entre os membros da família (verbal e não verbal), interesse, proximidade, acolhimento, comunicação, empatia, consistência de comportamentos e verbalizações e habilidade na resolução de problemas. A Adaptação familiar diz respeito à ausência de sentimentos e comportamentos negativos em relação à família, como raiva, incompreensão, além de competitividade entre os familiares. Por fim, a Autonomia familiar é a percepção 
que o indivíduo tem de sua família no aspecto autônomo, como confiança, privacidade e liberdade que cercam as relações familiares. As opções de resposta referem-se a "quase nunca ou nunca", "às vezes" e "quase sempre ou sempre", sendo representadas pelas pontuações 0,1 e 2 pontos, respectivamente. Quanto maior a pontuação, melhor a percepção de suporte familiar.

\section{Procedimento}

\section{Coleta de Dados}

Em ambas as amostras o instrumento foi aplicado de forma coletiva, nas salas de aula com a presença do professor de sala. Os alunos assinaram ao Termo de Assentimento Livre e Esclarecido (TALE), assim como seus responsáveis concordaram por meio da assinatura do Termo de Consentimento Livre e Esclarecido (TCLE). A coleta teve duração aproximada de 25 minutos em cada sala. A aplicadora realizou a leitura das instruções em voz alta, bem como ofereceu as devidas orientações de preenchimento.

\section{Análise de Dados}

Para estimação de evidências de validade com base na estrutura interna do IPSF empregou-se a análise fatorial exploratória (AFE), um procedimento que tem por objetivo a avaliação da estrutura de covariância entre um conjunto de variáveis, nesse caso, os itens do instrumento. Essa estrutura é interpretada em função de variáveis latentes, também denominados de fatores psicológicos, que determina a associação entre os itens (Izquierdo, Olea, \& Abad, 2014). A escolha de um procedimento exploratório para avaliação das propriedades psicométricas em uma nova população estão baseadas nas premissas de que esse procedimento, por permitir que todos os itens se correlacionem com todos os fatores, possibilita a compreensão da generabilidade da estrutura fatorial e também ajuda a identificar itens voláteis ou problemáticos para a população-alvo (Osborne \& Fitzpatrick, 2012).

Antes de submeter os dados à AFE, verificou-se os indicadores de adequação deles para a realização desse procedimento: índice de Kaiser-Meyer-Olkin (KMO) e Teste de esfericidade de Bartlett, tendo como referências valores superiores de $\mathrm{KMO}>0,7$ e teste de Bartlett significativos. Para indicação do número de fatores a serem retidos na estrutura fatorial, empregou-se o método Análise Paralela (AP) ou Parallel Analysis (PA), baseada no Minimum Rank Factor Analysis (Timmerman \& Lorenzo-Seva, 2011), a intepretação teórica do fator e a manutenção de itens com cargas fatoriais superiores a 0,30 .

A AFE foi realizada com método de estimação Unweighted Least Squares (ULS), e rotação oblíqua Promin (Lorenzo-Seva, 1999). Vale ressaltar que, em acordo com a natureza ordinal das variáveis (escala do tipo likert), todas as análises foram realizadas com base em matrizes de correlação policóricas (Izquierdo et al., 2014). Por fim, estimou-se indicadores de precisão para os fatores do IPSF por meio dos coeficientes alfa de Cronbach e ômega de McDonald. De acordo com a literatura, índices iguais ou superiores a 0,7 foram considerados adequados (Zumbo, Gadermann, \& Zeisser, 2007). Tais análises foram conduzidas com o programa Factor v. 10.10.01 (Ferrando \& Lorenzo-Seva, 2017).

Uma vez estimadas as evidências com base na estrutura interna e precisão da versão infanto-juvenil do IPSF (IPSF-IF), realizou-se procedimentos para obtenção de uma versão breve do instrumento. Para tanto, foram selecionados os seis itens com maiores cargas fatoriais em cada fator (haja vista a perspectiva de se contar com uma escala composta por menos de 20 itens). Essa versão foi aplicada em uma amostra independente de estudantes do $6^{\circ}$ ao $9^{\circ}$ ano do ensino fundamental, o que possibilitou a avaliação de suas propriedades psicométricas, conforme sugerido por Maiano et al. (2008). Para tanto, empregou-se a AFC, com método de estimação também adequado a natureza ordinal dos itens, Weighted Least Square (WLSMV) a partir da matriz de correlações policóricas. O modelo foi testado a partir dos índices recomendados por Muthén e Muthén (2012), são estes: $\mathrm{WLSMV} \chi^{2}, d f$, $\chi^{2} / d f$, RMSEA, CFI e TLI. Estabeleceu-se como parâmetro os seguintes valores para os índices de ajuste $\chi^{2} / d f<3$, RMSEA $<0,06$, CFI e TLI $>0,95$. A referida análise foi realizada por meio do programa MPlus 7.3 (Muthén \& Muthén, 2012).

\section{Considerações Éticas}

A pesquisa se respaldou na Resolução 466/2012 do Conselho Nacional de Saúde e seus complementares bem como foram seguidos todos os procedimentos éticos em conformidade com a Resolução 510/2016. Foi registrada e aprovada pelo Comitê de Ética em Pesquisa (CEP) de uma universidade pública, sob o $\mathrm{n}^{\circ}$ de parecer 2.125.734, CAAE: 68137417.3.0000.5231.

\section{Resultados}

Os resultados referentes ao índice $\mathrm{KMO}=$ 0,82309 e o teste de esfericidade de Bartlett's $=3870.3$ $(d f=861 ; p=0.000010)$, indicaram adequação da matriz de correlação para o uso de procedimentos de dimensionalidade. A partir dessa informação, realizou-se a Análise Paralela (AP) para indicação do número de fatores a serem retidos na solução fatorial. Os resultados evidenciaram a retenção de três, haja vista que apenas os três primeiros fatores estimados a partir dos dados reais apresentaram variância explicada $(26,5911,6.5246$ e 6,0035, respectivamente) superiores àquelas verificadas em média nas matrizes aleatórias $(5.2918,4.9624$ e 4.7294, respectivamente), bem como aos valores de variância explicada dos fatores estimados a partir das matrizes aleatórias alocados no percentil 95 (5.6833, 5.2352 e 4.9727, respectivamente). 
Considerando que a indicação do número de fatores correspondia à expectativa teórica que fundamentou o desenvolvimento da versão original do IPSF, a AFE foi realizada forçando-se uma estrutura de três fatores. Contudo, observou-se cinco itens que apresentaram cargas fatoriais inferiores a 0,30, a saber, itens: 2,12 ,
3031 e 41. Optou-se pela exclusão desses itens e realização de nova AFE, os resultados são apresentados na Tabela 1 onde é possível verificar as cargas fatoriais, porcentagem de variância explicada pelos fatores, índices de correlações entre os fatores e indicadores de precisão.

Tabela 1

Distribuição dos Itens por Fator e suas Respetivas Cargas Fatoriais

\begin{tabular}{|c|c|c|c|}
\hline \multirow{2}{*}{ Conteúdo dos itens } & \multicolumn{3}{|c|}{ Cargas Fatoriais } \\
\hline & 1 & 2 & 3 \\
\hline \multicolumn{4}{|l|}{ Fator 1: Autonomia Familiar } \\
\hline $\mathrm{I} 10$ & 0.565 & $-0,155$ & -0.128 \\
\hline I14 & 0.375 & -0.260 & 0.083 \\
\hline $\mathrm{I} 18$ & 0.356 & 0.101 & 0.101 \\
\hline I19 & 0.666 & 0.227 & -0.182 \\
\hline I34 & 0.641 & 0.122 & $-0,029$ \\
\hline I38 & 0.623 & -0.122 & 0.135 \\
\hline $\mathrm{I} 40$ & 0.472 & -0.019 & 0.079 \\
\hline \multicolumn{4}{|l|}{ Fator 2: Adaptação Familiar } \\
\hline I1 & 0.097 & 0.426 & -0.099 \\
\hline I6 & -0.124 & 0.431 & 0.134 \\
\hline I7 & 0.043 & 0.527 & 0.146 \\
\hline I8 & -0.161 & 0.633 & $-0,029$ \\
\hline I13 & 0.115 & 0.636 & -0.019 \\
\hline I16 & 0.078 & 0.575 & -0.109 \\
\hline $\mathrm{I} 20$ & -0.083 & 0.721 & -0.045 \\
\hline I21 & 0.230 & 0.488 & -0.048 \\
\hline $\mathrm{I} 27$ & -0.014 & 0.789 & -0.075 \\
\hline $\mathrm{I} 32$ & -0.068 & 0.576 & -0.138 \\
\hline 133 & 0.083 & 0.564 & -0.047 \\
\hline I36 & -0.049 & 0.583 & 0.136 \\
\hline I39 & 0.142 & 0.571 & 0.125 \\
\hline \multicolumn{4}{|l|}{ Fator 3: Afetivo-Consistente } \\
\hline I3 & -0.146 & -0.139 & 0.447 \\
\hline I4 & 0.056 & 0.131 & 0.362 \\
\hline I5 & -0.037 & -0.092 & 0.374 \\
\hline I9 & 0.047 & -0.231 & 0.629 \\
\hline I11 & -0.019 & -0.242 & 0.649 \\
\hline I15 & 0.026 & 0.053 & 0.480 \\
\hline I17 & 0.029 & -0.032 & 0.520 \\
\hline $\mathrm{I} 22$ & 0.080 & -0.059 & 0.560 \\
\hline $\mathrm{I} 23$ & 0.135 & -0.089 & 0.586 \\
\hline I24 & 0.115 & 0.068 & 0.645 \\
\hline I 25 & 0.136 & 0.058 & 0.650 \\
\hline I26 & 0.136 & 0.227 & 0.403 \\
\hline I28 & -0.004 & 0.272 & 0.442 \\
\hline I29 & -0.114 & -0.014 & 0.556 \\
\hline I35 & -0.073 & 0.109 & 0.709 \\
\hline I37 & -0.061 & 0.076 & 0.640 \\
\hline I42 & -0.024 & 0.025 & 0.620 \\
\hline
\end{tabular}


Tabela 1 (continuação)

Distribuição dos Itens por Fator e suas Respetivas Cargas Fatoriais

\begin{tabular}{|c|c|c|c|}
\hline \multirow{2}{*}{ Conteúdo dos itens } & \multicolumn{3}{|c|}{ Cargas Fatoriais } \\
\hline & 1 & 2 & 3 \\
\hline Porcentagem de variância explicada & 26,11 & 6,75 & 6,48 \\
\hline Total de variância explicada & & 39,34 & \\
\hline Correlação entre os fatores & F1 & F2 & F3 \\
\hline F1 & 1 & & \\
\hline F2 & 0.340 & 1 & \\
\hline F3 & 0.387 & 0.673 & 1 \\
\hline Alfa de Cronbach & 0.720 & 0.870 & 0.880 \\
\hline Ômega de McDonald & 0.726 & 0.871 & 0.881 \\
\hline
\end{tabular}

Conforme apresentado na Tabela 1, observa-se que o Fator 1 agrupa os itens originalmente desenvolvidos para avaliação da Autonomia familiar. Contudo, ressalta-se que neste estudo o fator deixou de contar com o item 12 (Minha família me faz sentir que posso cuidar de mim, mesmo quando estou sozinho(a)), excluído por não apresentar carga fatorial superior a 0,30 , passando assim a ser composto por sete itens. Quanto às cargas fatoriais, estas variaram entre 0,356 (item 18 - Em minha família existe privacidade) e 0,666 (item 19 - Minha família permite que eu seja do jeito que eu quero ser), demonstrando-se bons representantes do construto avaliado.

No Fator 2, foram agrupados os itens referentes a Adaptação familiar, ou seja, aqueles que expressam sentimentos negativos em relação a família. A análise sugeriu a manutenção de todos os 13 itens da versão original do IPSF. Quanto às cargas fatoriais, estas variaram entre 0,426 (item 1 - Acredito que a minha família tenha mais problemas emocionais que as outras famílias) e 0,789 (item 27 - Viver com minha família é desagradável), o que demonstra a adequação desses itens para a avaliação do referido fator.

O terceiro fator agrupou os itens cujo conteúdo se relacionava com expressões afetivas do contexto intrafamiliar, denominado Afetivo-Consistente. Esse fator foi composto por 17 itens, quatro a menos que a versão original haja vista suas exclusões por não apresentarem cargas fatoriais iguais ou superiores a 0,30 (os itens excluídos foram 2, 30, 31 e 41). Dessa forma, a variância total explicada pela estrutura fatorial foi de 39,34. Quanto à carga fatorial dos itens do fator, estas variaram entre 0,362 (item 4 - Meus familiares me elogiam) e 0,709 (item 35 - Em minha família demonstramos carinho por meio das palavras).

Já em relação à correlação entre os fatores, estas variaram entre moderada (Autonomia familiar e Adaptação familiar) e (Autonomia familiar e Afetivo-Consistente) e alta (Adaptação familiar e Afetivo-Consistente). Quanto aos indicadores de precisão dos fatores que compõem o IPSF, estes podem ser classificados como bom para o fator Autonomia familiar e desejáveis para os fatores Adaptação familiar e Afetivo-Consistente.

Na sequência, foi avaliada a estrutura interna e precisão da versão breve do IPSF-IJ. Os índices de ajuste indicaram adequação da referida versão, com valores classificados com muito bons: $\chi^{2}=230.055, d f=132$, $\chi^{2} / d f=1,74, p<0,001$ RMSEA $=0,046$ com IC $90 \%=0,036$ $-0,056, \mathrm{CFI}=0,956, \mathrm{TLI}=0,950 . \mathrm{Na}$ Tabela 2 , é possível verificar as cargas fatoriais dos itens, índices de correlação entre os fatores e indicadores de precisão.

Tabela 2

Modelo Fatorial Confirmatório da Versão Breve do IPSF-IJ

\begin{tabular}{|c|c|c|c|c|}
\hline & Itens* & AUT & ADP & AFT \\
\hline I1 & & 0.604 & & \\
\hline I2 & & 0.474 & & \\
\hline I3 & & 0.619 & & \\
\hline I4 & & 0.750 & & \\
\hline I5 & & 0.669 & & \\
\hline I6 & & 0.686 & & \\
\hline I7 & & & 0.645 & \\
\hline I8 & & & 0.683 & \\
\hline I9 & & & 0.687 & \\
\hline I10 & & & 0.822 & \\
\hline
\end{tabular}


Tabela 2 (continuação)

Modelo Fatorial Confirmatório da Versão Breve do IPSF-IJ

\begin{tabular}{|c|c|c|c|}
\hline Itens* & AUT & $\mathrm{ADP}$ & AFT \\
\hline I11 & & 0.829 & \\
\hline I12 & & 0.715 & \\
\hline I13 & & & 0.582 \\
\hline I14 & & & 0.466 \\
\hline I15 & & & 0.617 \\
\hline I16 & & & 0.714 \\
\hline I17 & & & 0.728 \\
\hline I18 & & & 0.775 \\
\hline Correlação entre os fatores & F1 & F2 & F3 \\
\hline F1 & 1 & & \\
\hline $\mathrm{F} 2$ & 0.086 & 1 & \\
\hline F3 & 0.387 & 0.550 & 1 \\
\hline Alfa de Cronbach & 0.878 & 0.805 & 0.779 \\
\hline Ômega de McDonald & 0.878 & 0.831 & 0.806 \\
\hline
\end{tabular}

Notas. *Itens com numeração sequencial de 1 a 18. AUT=Autonomia familiar; ADP=Adaptação familiar; AFT=Afetivo-Consistente

De acordo com a nova organização de itens, após as análises realizadas, foram verificados os resultados descritivos das amostras para o IPSF-IJ e para sua versão breve. A Tabela 3 apresenta a média de pontuação dos estudantes quanto às subescalas do instrumento de suporte familiar, o desvio padrão, a média ponderada de cada uma das subescalas (ou seja, a média de cada uma das subescalas foi dividida pelo número de itens, assim, pôde-se obter o resultado de predominância de cada uma delas, tendo em vista que as comparações foram feitas de maneira semelhante).

De acordo com dados descritivos apresentados na Tabela 3, pode-se observar que os estudantes apresentaram maiores médias na subescala Adaptação e menores médias no que tange a subescala Autonomia. Este e os demais resultados serão discutidos na sequência.

Tabela 3

Estatística Descritiva das Subescalas de Percepção de Suporte Familiar

\begin{tabular}{|c|c|c|c|c|c|c|c|c|}
\hline & \multicolumn{4}{|c|}{ Versão longa } & \multicolumn{4}{|c|}{ Versão breve } \\
\hline & M & $D P$ & $\begin{array}{c}\text { Média } \\
\text { Ponderada }\end{array}$ & $D P$ & M & DP & $\begin{array}{c}\text { Média } \\
\text { Ponderada }\end{array}$ & $D P$ \\
\hline Afetivo-Consistente & 21,04 & 6,58 & 1,24 & 0.39 & 7,69 & 2,93 & 1,28 & 0,48 \\
\hline Adaptação & 18,86 & 4,78 & 1,45 & 0.37 & 9,21 & 2,73 & 1,53 & 0,45 \\
\hline Autonomia & 7,54 & 7,54 & 1,07 & 0.39 & 5,74 & 2,83 & 0,96 & 0,47 \\
\hline Total & 47,65 & 11,45 & 1,29 & 0,31 & 22,65 & 5,92 & 1,51 & 0,39 \\
\hline
\end{tabular}

\section{Discussão}

Conforme exposto anteriormente, na versão da escala para crianças e adolescentes, após a retirada dos itens que não carregavam minimamente com 0,30 em algum dos fatores (cinco itens), a IPSF-IJ compôs-se por 37 itens. Dos itens retirados, quatro se referiam a subescala Afetivo-Consistente e um era da subescala Autonomia, sendo mantidos todos os itens da subescala Adaptação. Daqueles excluídos, por exemplo, o item 2 que diz: "As pessoas na minha família seguem as regras estabelecidas por eles", 30 "Em minha família há uma coerência entre as palavras e os comportamentos", 31 "Minha família sabe o que fazer quando surge uma emergência" e 41 "Meus familiares servem como bons modelos em minha vida", podem ter se apresentado um conteúdo mais complexo e/ou de difícil compreensão para escolares, sobretudo no ensino fundamental. Em consonância, o item 12 "Minha família me faz sentir que posso cuidar de mim, mesmo quando estou sozinho(a)", pode ter a interpretação de uma falta de suporte familiar, e não necessariamente de autonomia, segundo o fator no qual esse item estaria inserido.

No manual (Baptista, 2009), a amostra varia de 11 a 57 anos, não se referindo propriamente a escolares. Apresenta ainda média de idade de 22 anos e 6 meses, 
$(D P=7,1)$. Enquanto isso, o presente estudo foi composto apenas por escolares do ensino fundamental II, com idade média de 12 anos e 6 meses $(D P=1,2)$ para a primeira amostra e 12 anos e 3 meses para a segunda amostra $(D P=1,4)$. Assim, as adaptações realizadas, ao que parece, apresentam-se consistentes, tendo em vista que boa parte dos itens da escala se mantiveram e tiveram cargas fatoriais adequadas. Tais resultados sugerem as primeiras evidências de validade com base na estrutura interna para o IPSF-IJ frente uma amostra de crianças e adolescente, e assim o cumprimento do primeiro objetivo da presente pesquisa (AERA; APA, \& NCME, 2014). Já que os estudos de Costa et al. (2016) e Guidetti e Martinelli (2017), apenas verificaram evidências de validade baseadas na relação com outras variáveis.

Os resultados obtidos também permitem inferir a adequada estimação de evidências de precisão IPSF, uma vez que valores dos coeficientes empregados superiores a 0,7 podem ser considerados como bons indicadores de precisão de escalas de avaliação psicológica. Adicionalmente, os valores dos coeficientes alfa de Cronbach são coerentes com aqueles observados no manual do IPSF (Baptista, 2009). Tais resultados podem ser interpretados como a capacidade de os fatores mensurarem as respectivas variáveis psicológicas com baixo nível de erro assistemático associado à medida (Tabachnick \& Fidell, 2013). Além disso, indicam que foram estimadas as primeiras evidências de precisão do IPSF-IJ em uma amostra de crianças e adolescentes, também satisfatoriamente alcançados.

Ainda em relação à estrutura interna, destaca-se a replicação da estrutura fatorial composta por três fatores observada na presente pesquisa, bem como das poucas mudanças observadas no número de itens por fator quando comparado à versão original do IPSF, haja vista que o instrumentos teve suas primeiras evidências de validade estimadas por meio de Análise de Componentes Principais e baseadas em correlações de Pearson em seus estudos originais (Baptista, 2009). Dessa forma, poder replicar a estrutura por meio de métodos estatisticamente mais adequados às variáveis que compões IPSF, como AP e AFE baseadas em matrizes correlações policóricas, sugerem a adequação e a potencialidade de generalização da estrutura interna (Osborne \& Fitzpatrick, 2012).

Em relação às propriedades psicométricas da versão breve do IPSF-IJ, os resultados obtidos por meio métodos confirmatórios indicaram boas evidências de validade com base na estrutura interna, bem como indicadores de precisão desejáveis, ou seja, alfas superiores a 0,778 e ômegas superiores 0,806 (Tabachnick \& Fidell, 2013). Tais resultados corroboram a inferência de estabilidade da estrutura interna do instrumento quando avaliado por diferentes estratégias metodológicas. Adicionalmente, permite a inferência da estabilidade do instrumento ao avaliar diferentes amostras, haja vista que resultados foram obtidos junto a uma amostra de crianças independente daquela empregada na análise exploratória.

Ademais, ao disponibilizar uma versão breve do IPSF-IJ, cujas propriedades psicométricas sustentam a avaliação da percepção do suporte familiar, espera-se contribuir com pesquisadores da área. Sobretudo no que tange a possibilidade de aplicação do instrumento junto a verificação de outras variáveis, o que pode ser mais efetivo em uma versão breve, haja vista que, principalmente no caso de crianças e adolescentes, pode haver maior dispersão e desinteresse em responder a um número elevado de itens. Outro destaque é a aplicabilidade em contextos específicos, nos quais há a necessidade de um tempo reduzido para que a avaliação ocorra, como em ambientes escolares e hospitalares, por exemplo. (Serafini, Levandowski, \& Yates, 2018).

Os dados descritivos do IPSF-IJ e em sua versão breve indicaram resultados semelhantes. Em ambos os casos os estudantes apresentaram maiores médias na subescala Adaptação, seguido pela pontuação total, pela subescala Afetivo-Consistente e, por fim, menores médias no que tange a subescala Autonomia. Tendo em vista que, segundo Baptista (2009), a subescala Adaptação refere-se a comportamentos positivos em relação à família, tais como pouca ou nenhuma exclusão, vergonha, raiva, irritação, entre outros, os estudantes da amostra estudada conseguem identificar em suas famílias um funcionamento satisfatório no que tange a esses aspectos. Além disso, os resultados semelhantes em ambas as versões do instrumento sinalizam para uma consonância no que tange ao construto avaliado, com indicadores de evidências de validade com base na estrutura interna e precisão consideradas satisfatórias (Maiano et al., 2008; Marsh, Ellis, Parada, Richards, \& Heubeck, 2005; Smith et al., 2000).

Quanto às exclusões realizadas em relação ao manual (que foram cinco no total), hipotetiza-se que as subescalas Adaptação e Autonomia tangem comportamentos/afetos mais explícitos e claros para o aluno do ensino fundamental. Enquanto isso, a subescala AfetivoConsistente (que se refere à expressão de afetividade entre os membros da família, interesse, proximidade, acolhimento) inclui itens que demandam uma interpretação mais complexa por parte do respondente. Por exemplo, interesse e proximidade dos pais em relação aos filhos, podem ser compreendidos como desconfiança, ou superproteção, um entendimento característico da faixa de desenvolvimento da adolescência. É possível que os estudantes nessa faixa etária, correspondente ao início da adolescência, não tenham tanta flexibilidade emocional para reconhecer as questões afetivas despendidas por seus familiares (Barbosa \& Wagner, 2013).

Face às considerações trazidas, destaca-se a importância da realização dos estudos de evidências de validade com base na estrutura interna e precisão do IPSF para a população de escolares do ensino fundamental, 
considerando que essa faixa não se encontra totalmente contemplada na versão geral do instrumento de Baptista (2009). Além dos benefícios citados em relação à criação de uma versão reduzida para aplicação conjunta a outras variáveis e/ou em contextos específicos.

No que tange a essa faixa etária, cabe ressaltar a importância dos estudos sobre o suporte familiar, tendo em vista a relevância deste para o aumento do desempenho acadêmico e das demais competências desenvolvidas no contexto escolar, que começam a desenvolver-se ainda na fase inicial da escolarização, contemplada pelo ensino fundamental e posteriormente são aprimoradas no ensino médio (Batista et al., 2015; Benetti et al., 2016; Pozzobon \& Marin, 2017).

Cabe destacar, ainda, a relevância do estudo para amostras de crianças e adolescentes considerando que a percepção do suporte familiar pode trazer consequências ao seu desenvolvimento emocional. Com isso, aspectos positivos como a prevenção de distúrbios afetivos, uma maior autonomia e autoconceito podem ser considerados como fatores positivos e, como aspectos negativos, têm-se o estresse, a ansiedade e a depressão ligados à falta de um suporte familiar adequado (Costa et al., 2016; Gonçalves et al., 2016; Peñate, González-Loyola, \& Oyanadel, 2020; Van Harmelen et al., 2016). Essas associações com variáveis externas devem ser pesquisadas em estudos futuros.

Há que se considerar as limitações encontradas no presente estudo. Destaca-se que este fora realizado com uma amostra de escolares de um único estado, sendo fundamental o emprego de futuras pesquisas que busquem abarcar tal temática em outras realidades e amostras. Além disso, sugere-se a realização de estudos com amostras maiores de estudantes do ensino fundamental, visando a criação de normas para essa faixa etária, além da realização de estudos relacionando à percepção de suporte familiar com variáveis psicoeducacionais, tais como a motivação para aprender, as estratégias de aprendizagem, a compreensão de leitura, entre outras. Adicionalmente, sugere-se a realização de estudos que avaliem a estabilidade entre as versões longas e curtas ao se relacionarem com essas variáveis externas.

\section{Agradecimentos}

Não constam agradecimentos.

\section{Financiamento}

A presente pesquisa não recebeu nenhuma fonte de financiamento sendo custeada com recursos dos próprios autores.

\section{Contribuição dos autores}

Declaramos que todos os autores participaram da elaboração do manuscrito. Especificamente, o(s) autor(es) Makilim, Katya, Amanda e Marcella participou(ram) da redação inicial do estudo - conceitualização, investigação, visualização, o(s) autor(es) Makilim, Katya e Evandro participou(ram) da análise dos dados, e o(s) autor(es) Makilim, Katya, Amanda e Evandro participou(ram) da redação final do trabalho - revisão e edição

\section{Disponibilidade dos dados e materiais}

Todos os dados e sintaxes gerados e analisados durante esta pesquisa serão tratados com total sigilo devido às exigências do Comitê de Ética em Pesquisa em Seres Humanos. Porém, o conjunto de dados e sintaxes que apoiam as conclusões deste artigo estão disponíveis mediante razoável solicitação ao autor principal do estudo.

\section{Conflito de interesses}

Os autores declaram que não há conflitos de interesses.

\section{Referências}

American Educational Research Association, American Psychological Association, \& National Council on Measurement in Education. (2014). Standards for educational and psychological testing. Washington, DC: Autor.

Baptista, M. N. (2005). Desenvolvimento do Inventário de Percepção de Suporte Familiar (IPSF): estudos psicométricos preliminares. Psico-USF, 10(1), 11-19. Recuperado de http://pepsic.bvsalud.org/pdf/psicousf/v10n1/v10n1a03.pdf

Baptista, M. N. (2007). Inventário de percepção de suporte familiar (IPSF): estudo componencial em duas configurações. Psicologia: Ciência e Profissão, 27(3), 496-509. doi:10.1590/S1414-98932007000300010

Baptista, M. N. (2009). Inventário de percepção de suporte familiar - IPSF. São Paulo: Vetor.

Baptista, M. N., Rueda, F. J. M., \& Brandão, E. M. (2017). Suporte familiar e autoconceito infantojuvenil em acolhidos, escolares e infratores. Psicologia em Pesquisa, 11(1), 55-64. doi:10.24879/2017001100100212

Barbosa, P. V., \& Wagner, A. (2013). A autonomia na adolescência: Revisando conceitos, modelos e variáveis. Estudos de Psicologia, 18(4), 649658. doi:10.1590/S1413-294X2013000400013

Bastos, D. S., Scortegagna, S. A., Baptista, M. N., \& Cremasco, G. S. (2016). Sintomas depressivos e suporte familiar em idosos e adultos em hemodiálise. Psicologia Teoria e Prática, 18(2), 103-116. doi:10.15348/1980-6906/psicologia.v18n2p103-116

Batista, E. C., Mantovani, L. K. S., \& Nascimento, A. B. (2015). Percepção de suporte familiar de alunos com histórico de reprovação escolar. Debates em Educação, 7(13), 50-70. doi:10.28998/2175-6600.2015v7n13p50

Benetti, I. C., Vieira, M. L., \& Faracco, A. M. (2016). Suporte parental para crianças do ensino fundamental. Cadernos de Pesquisa, 46(161), 784-801. doi:10.1590/198053143424

Castro, M. C. D., \& Cruz, R. M. (2015). Prevalência de transtornos mentais e percepção de suporte familiar em policiais civis. Psicologia: Ciência e Profissão, 35(2), 271-289. doi:10.1590/1982-370300702013 
Costa, K., Montiel, J. M., Bartholomeu, D., Murgo, C. S., \& Campos, N. R. (2016). Percepção do suporte familiar e desempenho em leitura e escrita de crianças do ensino fundamental. Revista Psicopedagogia, 33(101), 154-163. Recuperado de http://pepsic.bvsalud.org/scielo. php?script $=$ sci arttext\&pid $=$ S0103-84862016000200005

Ferrando, P.J., \& Lorenzo-Seva, U. (2017). Program FACTOR at 10: origins, development and future directions. Psicothema, $29(2), 236-241$. doi: $10.7334 /$ psicothema2016.304

Gonçalves, M., Baptista, M. N., \& Farcas, D. (2016). IPSF: Análise da estrutura interna em uma amostra de jovens adultos portugueses. Avaliação Psicológica, 15(1), 115-123. Recuperado de http://pepsic.bvsalud.org/scielo.php?script=sci_arttext\&pid=S1677$04712016000100013 \& \operatorname{lng}=\mathrm{pt} \& \mathrm{l} \operatorname{lng}=\mathrm{pt}$

Guidetti, A. A., \& Martinelli, S. C. (2017). Percepções infantis: Relações entre motivação escolar e suporte familiar. Psico-USF, 22(3), 515-525. doi:10.1590/1413-82712017220311

Izquierdo, I., Olea, J., \& Abad, F. J. (2014). Exploratory factor analysis in validation studies: Uses and recommendations. Psicothema, 26(3), 395-400. doi: 10.7334/psicothema2013.349

Knight, C. J., Boden, C. M., \& Holt, N. L. (2010). Junior tennis players' preference for parental behaviours. Journal of Applied Sport Psychology, 22(4), 377-391. doi:10.1080/10413200.2010.495324

Lorenzo-Seva, U. (1999). Promin: A method for oblique factor rotation. Multivariate Behavioral Research, 34, 347-356. doi:10.1207/ s15327906mbr3403_3

Maiano, C., Morin, A. J. S., Ninot, G., Monthuy-Branc, J., Stephan, Y., Florent, J., \& Vallée, P. (2008). A short and very short form of the physical self-inventory for adolescents: Development and factor validity. Psychology of Sport and Exercise 9(6), 830-847. doi:10.1016/j. psychsport.2007.10.003

Marsh, H. W., Ellis, L., Parada, R., Richards, G. E., \& Heubeck, B. (2005). A short version of the Self-Description Questionnaire II: Operationalizing criteria for short-form evaluation with new applications of confirmatory factor analyses. Psychological Assessment, 17(1), 81-102. doi:10.1037/1040-3590.17.1.81

Muthén, L. K., \& Muthén, B. O. (2012). Mplus user's guide (7th ed.). Los Angeles: Muthén \& Muthén.

Osborne, J., \& Fitzpatrick, D. (2012). Replication analysis in exploratory factor analysis: What it is and why it makes your analysis better. Practical Assessment Research \& Evaluation, 17(15), 1-8. doi:10.7275/h0bd-4d11

Peñate, W., González-Loyola, M., Oyanadel, C. (2020) The Predictive Role of Affectivity, Self-Esteem and Social Support in Depression and Anxiety in Children and Adolescents. International Journal of Environmental Research and Public Health, 17, 6984. doi: 10.3390/ ijerph17196984

Pozzobon, M., \& Marin, A. H. (2017). Suporte familiar e metas profissionais de adolescentes com baixo desempenho escolar. Investigação Qualitativa em Ciências Sociais, 3, 1-7. Recuperado de https://proceedings.ciaiq.org/index.php/ciaiq2017/article/viewFile/1429/1386

Ribeiro, R., Ciasca, S. M., \& Capelatto, I. V. (2016). Relação entre recursos familiares e desempenho escolar de alunos do $5^{\circ}$ ano do ensino fundamental de escola pública. Revista psicopedagogia, 33(101), 164-174. Recuperado de http://pepsic.bvsalud.org/pdf/psicoped/ v33n101/06.pdf

Roksa, J., \& Kinsley, P. (2019). Correction to: The Role of Family Support in Facilitating Academic Success of Low-Income Students. Research in Higher Education, 60(437). doi:10.1007/s11162-018-9525-z

Serafini, A. J., Levandowski, D. C., \& Yates, D. B. (2018). Os testes psicométricos na avaliação com crianças. Em M. C. Lins, M. Muniz \& L. Moraes. (Eds.). Avaliação Psicológica Infantil (pp. 229-243). São Paulo: Hogrefe.

Smith, G., McCarthy, D., \& Anderson, K. (2000). On the sins of short-form development. Psychological Assessment, 12(1), 102-111. doi: 10.1037/1040-3590.12.1.102

Tabachnick, B. G., \& Fidell, L. S. (2013). Using Multivariate Statistics. Boston, MA Pearson.

Timmerman, M. E., \& Lorenzo-Seva, U. (2011). Dimensionality assessment of ordered polytomous items with parallel analysis. Psychological Methods, 16(2), 209-220. doi:10.1037/a0023353

Van Harmelen, A. L., Gibson, J. L., St Clair, M. C., Owens, M., Brodbeck, J., Dunn, V., Lewis, G., Croudace, T., Jones, P. B., Kievit, R.A., \& Goodyer, I. M. (2016). Friendships and family support reduce subsequent depressive symptoms in at-risk adolescents. PLoS ONE, 11(5). doi:10.1371/journal.pone.0153715

Zumbo, B. D., Gadermann, A. M., \& Zeisser, C. (2007). Ordinal Versions of Coefficients Alpha and Theta for Likert Rating Scales. Journal of Modern Applied Statistical Methods, 6(1), 21-29. doi:10.22237/jmasm/1177992180

\section{Sobre os autores}

Makilim Nunes Baptista é Psicólogo, doutor em Ciências pela Universidade Federal de São Paulo e docente do Programa de PósGraduação Stricto Sensu em Psicologia da Universidade São Francisco-Campinas/SP. Bolsista Produtividade Nível 1C/CNPq.

Katya Luciane de Oliveira é Psicóloga, doutora pela Unicamp e docente do Programa de Mestrado e Doutorado em Educação e do Programa de Pós-Graduação em Psicologia ambos da Universidade Estadual de Londrina. Bolsista Produtividade Nível 2/CNPq.

Amanda Lays Monteiro Inácio é Psicóloga (UEL), doutoranda do Programa de Pós-graduação Stricto Sensu em Psicologia da Universidade São Francisco. Docente do curso de Psicologia da Universidade Estadual de Londrina.

Marcella das Neves Burgos é Psicóloga (UEL), mestre em Educação pelo Programa de Mestrado e Doutorado em Educação da Universidade Estadual de Londrina.

Evandro Morais Peixoto é Docente do Programa de Pós-Graduação Stricto Sensu em Psicologia da Universidade São Francisco. Doutor em Psicologia pela PUC/Campinas. Membro do GT Avaliação Psicológica em Psicologia Positiva e Criatividade na ANPEPP.

\section{Como citar este artigo}

Baptista et al. (2020). Versão Infantojuvenil do Inventário de Percepção de Suporte Familiar (IPSF-IJ). Avaliação Psicológica, 19(4), 441-450. http://dx.doi.org/10.15689/ap.2020.1904.18533.10 